\title{
IIRSA, subimperialismo brasileño y resistencias populares. Los conflictos en torno a la represa de Inbambari (Perú) y de la carretera sobre el TIPNIS (Bolivia)
}

\author{
IIRSA, Brazilian sub-imperialism and popular resistance. Conflicts around the Inbambari \\ dam (Peru) and the highway over the TIPNIS (Bolivia)
}

\begin{abstract}
Mariano Féliz
Doctor en Economía. Doctor en Ciencias Sociales. Centro de Investigaciones Geográficas (CIG). Instituto de Investigaciones en Humanidades y Ciencias Sociales (IdIHCS/CONICET). Universidad Nacional de La Plata (UNLP). Calle 51 e/124 y 125. Oficina C313-C315, Edificio C (1925) Ensenada, Buenos Aires, Argentina, marianfeliz@gmail.com, ORCID https://orcid.org/0000-0002-3000-3873

Daiana Melón

Doctoranda en Ciencias Sociales (UNLP). Becaria doctoral de la Universidad Nacional de La Plata UNLP. Centro de Investigaciones Geográficas (CIG). Instituto de Investigaciones en Humanidades y Ciencias Sociales (IdIHCS/CONICET). Universidad Nacional de La Plata (UNLP). Calle 51 e/124 y 125. Oficina C313-C315, Edificio C (1925) Ensenada, Buenos Aires, Argentina, daianamelon@gmail.com, ORCID https://orcid.org/0000-0003-2074-0201
\end{abstract}

Recibido: 5 de febrero 2020 || Aprobado: 16 de junio 2020

Resumen

Este artículo aborda la forma en que el proyecto brasileño de desarrollo dependiente se desenvuelve en la constitución y consolidación de la Iniciativa para la Integración de la Infraestructura Regional Suramericana (IIRSA). Retomando los postulados de la teoría de la dependencia, específicamente los de Marini, se analizan las tendencias subimperialistas emanadas de Brasil, a partir de la promoción de un tipo de integración y una división regional del trabajo al servicio de los intereses de su burguesía. Así, se realiza un análisis de la IIRSA como etapa reciente de las estrategias de integración sudamericana. A su vez, se discute de qué forma la estrategia subimperialista brasileña y las resistencias populares se manifiestan en la Iniciativa a través de dos proyectos de inversión: la represa Inambari en Perú y la carretera sobre el Territorio Indígena y Parque Nacional Isiboro Secure en Bolivia.

Palabras clave: Subimperialismo; Brasil; Resistencias socioterritoriales; Integración regional

Abstract

This article addresses the way in which the Brazilian project of dependent development unfolds in the constitution and consolidation of the Initiative for the Integration of the Regional Infrastructure of South America (IIRSA). Taking up the postulates of dependency theory, specifically those of Marini, the subimperialist tendencies emanating from Brazil are analyzed, based on the promotion of a type of integration and a regional division of labor at the service of the interests of its bourgeoisie. Thus, an analysis of the IIRSA is carried out as a recent stage of South American integration strategies. In turn, it is discussed how the Brazilian sub-imperialist strategy and popular resistance are manifested in the Initiative through two investment projects: the Inambari dam in Peru and the highway over the Isiboro Secure Indigenous Territory and National Park in Bolivia.

Key words: Subimperialism; Brazil; Socioterritorial resistances; Regional integration

Cita sugerida: Féliz, M. y Melón, D. (2020). IIRSA, subimperialismo brasileño y resistencias populares. Los conflictos en torno a la represa de Inbambari (Perú) y de la carretera sobre el TIPNIS (Bolivia). Estudios Socioterritoriales. Revista de Geografía, (28), 056. DOI: https://doi.org/10.37838/unicen/est.28-056 


\section{INTRODUCCIÓN}

En las décadas de 1960 y 1970, los debates en torno a las estrategias de integración regional y los límites del desarrollo capitalista en América Latina tuvieron un salto cualitativo. En su contienda con el estructuralismo, los postulados que se impulsaron desde la Comisión Económica para América Latina y el Caribe (CEPAL) y el proyecto imperialista de Estados Unidos, la teoría de la dependencia vino a aportar nuevas hipótesis, en especial su vertiente marxista encabezada por Ruy Mauro Marini (1970). Este autor impulsó la idea del surgimiento en la región de tendencias subimperialistas en algunos países. En particular, Brasil aparecía como principal eje articulador de estas tendencias en Sudamérica. Este cambio modificaría radicalmente la lógica y fundamentos de las estrategias de integración regional. Así, Brasil promovería un tipo de integración y una división regional del trabajo al servicio de los intereses de su burguesía.

A cuatro décadas del inicio de este debate, las tensiones subimperialistas emanadas de Brasil han sufrido un desarrollo novedoso que opera en el marco de la conformación y desenvolvimiento de la Iniciativa para la Integración de la Infraestructura Regional Suramericana (IIRSA). Nacida en el 2000, en 2009 la IIRSA quedó bajo la órbita de la Unión de Naciones Suramericanas (UNASUR), a través del Consejo Suramericano de Infraestructura y Planeamiento (COSIPLAN), donde la IIRSA aparecía como foro técnico. Esta proyecta de manera parcial, contradictoria y disputada, el modelo de desarrollo capitalista de Brasil como potencia subimperialista regional. En particular, tiene como uno de sus principales ejes de articulación la integración energética, eje fundamental de la expansión brasileña.

En este trabajo indagaremos en la forma en que el proyecto brasileño de desarrollo dependiente se desenvuelve en el marco de la constitución y consolidación del COSIPLAN-IIRSA. Para ello, recuperaremos el debate sobre el subimperialismo como categoría teórica para comprender la forma del desarrollo capitalista dependiente en la región, partiremos del debate clásico y retomaremos los planteos contemporáneos. En particular, buscaremos incorporar la discusión sobre las tensiones que atraviesan el proyecto subimperialista, la significación que tienen en su seno los proyectos energéticos y las oposiciones que suscita y que lo ponen en cuestión. En segundo lugar, abordaremos el análisis de la Iniciativa como etapa más reciente de las estrategias de integración en la región suramericana. En tercer lugar, discutiremos de qué forma la estrategia subimperialista de Brasil y las resistencias populares a la misma se manifiestan en el seno de la Iniciativa a través de dos proyectos de inversión en infraestructura regional prototípicos: la represa hidroeléctrica de Inambari en Perú, y la carretera sobre el Territorio Indígena y Parque Nacional Secure (TIPNIS) en territorio boliviano. Finalmente, presentaremos algunas conclusiones preliminares que señalan las principales contradicciones de la IIRSA en tanto expresión del proyecto del capitalismo brasileño.

\section{LA HIPÓTESIS SUBIMPERIALISTA}

El marxismo latinoamericano en la vertiente dependentista propone un análisis del desarrollo del capitalismo en la región que permite explicar, por un lado, la reproducción ampliada de la dependencia y, al mismo tiempo, el desarrollo de formaciones sociales particulares que se desprenden del conjunto para convertirse en potencias subimperialistas sin por ello superar su carácter dependiente (Marini, 1970). El subimperialismo es una formación social particular que nace del seno de la economía dependiente (Luce, 2015). En tal sentido, es un nivel jerárquico del sistema mundial capitalista y, al mismo tiempo, es la etapa superior del capitalismo dependiente, en la cual algunas formaciones socioeconómicas se ven transformadas en nuevos eslabones en la cadena de las potencias imperialistas (Luce, 2015).

\section{DEPENDENCIA Y RESISTENCIA}

El capitalismo latinoamericano se constituyó a partir de la conquista de América por parte de 
las potencias europeas en transición al capitalismo. Ese choque colocó a América Latina como proveedor de alimentos e insumos (materias primas) que fortalecieron en el centro la progresiva y orgánica transición del predominio de formas de explotación basadas en estrategias de plusvalía absoluta a modalidades sostenidas crecientemente en formas de plusvalía relativa (Marini, 2015). Estas últimas suponían la transición a formas de producción apoyadas en modalidades más intensivas en capital constante. En esos espacios nacionales de valor, permitieron una integración más orgánica de la fuerza de trabajo como capital variable. Esto habilitó un mayor desarrollo de la productividad del trabajo en el centro y, en consecuencia, la consolidación de modalidades de articulación en el ciclo global del capital desde posiciones de ventaja absoluta dinámica.

En esa articulación de países centrales y dependientes, América Latina quedó mayormente encerrada en una modalidad de desarrollo del capital sostenida en formas de producción de plusvalía absoluta. Esta situación es producto de la necesidad de los territorios dependientes de compensar internamente las pérdidas sistemáticas de valor y plusvalor en el intercambio desigual con los países centrales (Marini, 2015; Osorio, 2017; Féliz y Haro, 2019).

La paradoja es que en estos espacios de valor dependientes, las formas de explotación del trabajo y la naturaleza son, simultáneamente, más brutales e inestables. El capital dependiente tiene enormes dificultades para imponerse en la producción a través de la forma impersonal de la mercancía y su fetiche, es decir, a través de la mecanización de los procesos de trabajo y mercantilización de las relaciones sociales y con la naturaleza. En consecuencia, privilegian formas más intensivas en explotación del trabajo vivo. Mientras en los países centrales el capital constante opera como medio dominante para el control de los procesos de trabajo y valorización, en la economía dependiente las dificultades para acumular conducen a una dinámica diferente (Caffentzis, 2013). Aquí, el control social del trabajo se realiza más intensamente a través de la coacción directa o por la vía de formas más extendidas de precarización del empleo, es decir a través de la superexplotación de la fuerza de trabajo.

Las consecuencias de la dependencia son múltiples, pero la central es que se convierte en una limitante estructural para la mejora sostenida de las condiciones de vida de la población en relación a las posibilidades materiales de la sociedad. La reproducción ampliada del ciclo del capital en la economía dependiente restringe de manera extraordinaria las posibilidades de expansión de las condiciones de reproducción de la fuerza de trabajo (Marini, 2015).

Esto supone que la lucha de clases y las formas del conflicto operan en la economía dependiente dentro de determinaciones particulares que son distintas de aquellas que prevalecen en el centro. Aquí, la resistencia obrera en el plano de la explotación directa del trabajo se manifiesta en modalidades menos mediadas por la forma estatal (o medidas en formas más inestables), y, por tanto, donde la acción directa y el enfrentamiento cara-a-cara con el capital es más evidente; de allí la naturaleza 'explosiva' de la conflictividad social. En el plano de la reproducción, el capital en la economía dependiente confronta al trabajo reproductivo y de cuidados -centrado en la explotación de las mujeres- con mayores grados de dificultad para imponer el patriarcado del salario (Federici, 2018; Féliz y Díaz Lozano, 2018). En el conjunto de América Latina, predominan formas de organización familiar que aún hoy oponen obstáculos -no absolutos- al desarrollo de las formas capitalistas, incluso dentro de formaciones patriarcalizadas (Cielo y Vega, 2015). Finalmente, la irrupción del capital imperialista, con el fin de avanzar en modalidades de saqueo de los bienes comunes, choca con prácticas de vida que operan formas más orgánicas de interacción de las comunidades con/en la naturaleza (Féliz y Migliaro, 2017).

\section{DE LA DEPENDENCIA AL SUBIMPERIALISMO (DEPENDIENTE)}

En este marco, determinados países pueden conseguir superar algunos umbrales en la acu- 
mulación de capital, llevando el nivel de su composición orgánica -que podemos aproximar a través del valor del capital constante por trabajador- a valores suficientes como para convertirse en centros de producción y exportación regional de capital, aun dentro de la dependencia. Esos territorios se convierten en punto de apoyo de estrategias subimperialistas (Marini, 1970). Para ello, esos países deben poder superar las barreras, aunque no los límites, que impone el conflicto de clases a escala local en su articulación como parte del conflicto a escala global. Los espacios nacionales subimperialistas deben alcanzar: (a) un nivel intermedio de composición orgánica del capital en el sistema productivo local en la escala global; y (b) la capacidad de ejercer una política expansionista autónoma acompañada por una mayor integración al ciclo global del capital imperialista, pero manteniéndose dentro del marco ejercido por él (Marini, 1977).

En este marco, las potencias subimperialistas traducen sus contradicciones internas de clase en una acción expansionista regional. Al ampliar los parámetros de la acumulación de capital constante fijo por encima de la media regional, las tendencias a la expansión del mercado impulsadas por la propia acumulación son desplegadas en los espacios de valor inmediatos. En América Latina, en relación al avance de la industrialización, unos pocos países lo han conseguido en mayor o menor medida: Brasil, y con mucho menor alcance Argentina y México. Un estudio realizado por Aguiar de Medeiros (1986) analiza los niveles de inversión en sectores industriales en Brasil entre 1969 y 1979: la inversión en el sector metalúrgico pasó de 53.1 millones de dólares en 1969 a 1160.5 en 1979; el mecánico pasó de 18.8 a 122.7; el de material eléctrico y comunicaciones de 19.9 a 174.5; el de materiales de transporte de 131.4 a 567.1 ; y el de productos químicos de 72.2 a 723.4 ; concluyendo que el total de la inversión en manufacturas pasó de 544.1 a 3861.8 millones de dólares. A su vez, mientras que en el resto de los países sudamericanos el porcentaje de exportación de manufacturas, entre 1965 y 1985, se mantuvo constante (no superando ningún país los 10 puntos), en Brasil este porcentaje pasó de 2.1 en 1965 a 28.4 en 1985 (Fajnzylber, 1990).

A partir de esa posición, algún país puede convertirse en potencia subimperialista si -en paralelo- consigue imponer una división del trabajo a escala subregional en función de los intereses de su burguesía local. En ese proceso, el capital de la potencia subimperialista logra compensar parcialmente las pérdidas de plusvalía en la competencia con los países centrales imperialistas, a partir de (a) la superexplotación de su propia fuerza de trabajo; y (b) la apropiación de valor producido en otros espacios nacionales a partir de un proceso específico, regional, de intercambio desigual (Luce, 2015). Asimismo, el desarrollo del subimperialismo supone la conversión del espacio regional en territorio para la expropiación y saqueo a escala ampliada de las riquezas naturales. En la misma medida en que Rosa Luxemburg analizó que el proceso de acumulación originaria de capital era permanente y algo inherente al expansionismo imperialista (Luxemburg, 2018), entendemos que el subimperialismo asume ese carácter a través de formas de saqueo recurrente de los territorios circundantes.

En América Latina, solo Brasil habría conseguido históricamente ubicarse en este nuevo plano. La dictadura de 1964 se convirtió en el punto de quiebre que abrió con claridad el proceso transicional que permitiría a la burguesía local desarrollar una estrategia integral a escala regional, en cooperación antagónica con las fuerzas del capital imperialista (Marini, 1970).

Marini retomó la noción de cooperación antagónica de August Thalheimer, quien planteó que el proceso de expansión imperialista implica la formación de polos económicamente pujantes donde penetran los capitales de las economías centrales y que se expanden hacia otras partes del mundo (Clemente, 2018). Recuperando este concepto, Luce (2007) postula que el Estado brasileño estableció una relación de cooperación antagónica con la potencia dominante, con el fin de imprimir una política expansionista relativamente autónoma. Esta diná- 
mica se instauró en la política exterior, así como en las contradicciones de clase de la sociedad dependiente. Esta relación era autónoma en el sentido de constituir una esfera de influencia regional propia, vinculada a los intereses de fracciones nacionales del capital. Se estableció una división regional del trabajo en la que Brasil compraba a los países materias primas y les vendía manufacturas. Sin embargo, esta fue relativamente autónoma, dado que en otras áreas de la política internacional Brasil siguió el anticomunismo estadounidense. La burguesía brasileña se encontraba en una posición de cooperación antagónica con su par estadounidense, dado que mientras se impulsaba una reestructuración interna que integrase a Brasil al nuevo orden económico y se profundizaba la dependencia de la economía hegemónica, en su intento de obtener mayores ventajas chocaba con Estados Unidos (Clemente, 2018).

\section{SUBIMPERIALISMO, ESTADO E INFRAESTRUCTURA ENERGÉTICA}

La dinámica del desarrollo del subimperialismo brasileño asume un doble juego: (a) la configuración regional de la forma del Estado dependiente suramericano como expresión de la expansión subimperialista del capital en Brasil; y (b) la necesidad de articular las condiciones materiales para el desenvolvimiento de la valorización de ese capital a escala regional. En este proceso, el desarrollo del Estado capitalista dependiente se configura para constituir una estrategia de expansión regional para el capital brasileño. Este movimiento asume un plano de orden militar-institucional y otro de integración infraestructural y financiero. En el primer plano, Brasil ha construido una estrategia de control del 'espacio vital' de sus capitales subimperialistas a los fines de garantizar formas de dominio del territorio regional. En el segundo plano, Brasil ha promovido estrategias de integración regional que le garanticen (aunque sea de manera disputada y contradictoria) el desarrollo y control de la infraestructura regional disponible para el saqueo de los bienes comunes (incluida las que contienen energía potencial) y el flujo de mercancías hacia los mercados regionales e internacionales.

Es en esta última dimensión que le cabe un rol central en la era contemporánea a la IIRSA. La misma expresa, de manera conflictiva, los intereses expansionistas del gran capital brasileño en su intento de consolidar una posición de articulación entre las exigencias del imperialismo norteamericano y europeo, y las de la burguesía local. Tales intenciones son limitadas por la resistencia de las comunidades y pueblos cuyas prácticas de reproducción vital son puestas en cuestión por los proyectos de infraestructura que la IIRSA propone. Rosa Luxemburg (2018) explicó la centralidad de este movimiento destructivo en las experiencias imperialistas. La proyección subimperialista opera en la misma dirección, aunque en otra escala.

El desarrollo del capital a nivel nacional e internacional supone la capacidad de contener e integrar la resistencia popular frente a la destrucción de las formas de reproducción de la vida y su cosificación (Cleaver, 1985). En el mismo sentido, el avance subimperialista implica que el capital en expansión busca crear las condiciones para imponer sus exigencias. En el caso de Brasil, la ampliación de la base regional de explotación supone construir vías de transporte de mercancías y, en especial, nuevos proyectos energéticos.

Son precisamente estos proyectos de apropiación capitalista de las formas de energía potencial acumulada en la naturaleza uno de los puntos más frágiles del proyecto subimperialista brasileño. La configuración de ratios específicos de trabajo/energía son claves para comprender la forma en que se organizan patrones particulares de reproducción capitalista (Caffentzis, 1980). El avance en la composición orgánica del capital en un territorio supone tanto el desplazamiento de trabajo vivo por trabajo muerto, como el desplazamiento de energía humana (trabajo) por formas de energía no humanas.

El desarrollo de ratios trabajo/energía apropiados a las formas de explotación contemporánea en el marco de la dependencia requiere para 
la potencia subimperialista el control del territorio adyacente. La expansión subimperialista del Brasil remite a la imperiosa necesidad del capital nacional brasileño de apropiar energía (en particular, en la forma de energía eléctrica) a partir de la destrucción de los territorios aledaños. El expansionismo subimperialista sobre los recursos energéticos potenciales incluyen el desarrollo de infraestructura para el saqueo de los sustratos materiales con hidrocarburos (en el caso de Brasil, por ejemplo, el proyecto Pre-Sal en la plataforma marina) o la quema de bosques nativos para el avance de las plantaciones destinadas a los agrocombustibles. El avance de la integración regional es, en este sentido, también el desarrollo de formas de producción de energía eléctrica basadas en la transformación de la energía potencial en los ríos, sobre el sustrato de la destrucción de las formas de vida natural y comunidades que ocupan los territorios a la vera de los mismos. Los proyectos de Inambari y el TIPNIS -que analizaremos más adelante- son, en esa clave, ejemplos sustantivos de las tensiones y complejidades del proyecto subimperialista.

\section{TRANSFORMACIONES DE LA INTEGRACIÓN REGIONAL EN SUDAMÉRICA}

Como ya señalamos, el COSIPLAN-IIRSA aparece como la etapa superior del proyecto de integración subimperialista de Brasil. No es la primera estrategia ni será, seguramente, la última iniciativa.

A partir de la década de 1930, las guerras mundiales y la crisis de la división internacional del trabajo crearon una coyuntura que inauguró la posibilidad de desarrollar cambios en las economías latinoamericanas. Aquellos países que habían constituido un embrión de mercado interno y un desarrollo de las fuerzas productivas iniciaron un proceso de industrialización por sustitución de importaciones, lo que conllevó el ingreso de multinacionales portadoras de tecnología (Bambirra, 1995). Sin embargo, la estrechez de la capacidad de consumo resultante de las presiones de la dependencia puso en jaque la continuidad de este proceso (Marini, 2015). En la década de 1960, las exigencias de las transnacionales en términos de escalas mínimas de producción pusieron la integración latinoamericana en la agenda; a estas presiones se sumaron las tensiones en el capitalismo dependiente brasileño que se convertirían -luegoen tendencias subimperialistas (Marini, 1970). En este marco, y retomando las ideas impulsadas por Raúl Prebisch desde la CEPAL ${ }^{1}$, se constituyeron dos iniciativas de integración económica: la Asociación Latinoamericana de Libre Comercio (ALALC) y el Grupo Andino (de Arruda Sampaio Jr., 2008). Los países integrantes de la ALALC fueron Bolivia, Colombia, Ecuador, Perú y Venezuela -quienes formaban parte de la Comunidad Andina-, Argentina, Brasil, Paraguay, Uruguay, Chile y México. En 1980, esta instancia fue reemplazada por la Asociación Latinoamericana de Integración. Con este cambio, el objetivo de alcanzar una zona regional de libre comercio fue reconvertido bajo la idea de un mercado común (Gil y Paikin, 2013).

Las ideas que se impulsaron desde la CEPAL allanaron el camino para las reformas de mercado implementadas durante la década de 1980 en la región (bajo la marca de la 'transformación productiva con equidad' y el 'regionalismo abierto'), las cuales transformaron profundamente la estructura política, económica y social de los países latinoamericanos (Gudynas, 2006).

Desde inicios de la década de 1990 el presidente brasileño Itamar Franco en Brasil postuló la idea de la creación de un área de libre comercio sudamericana. Sin embargo, esta no fue acompañada por el resto de los países de la región (Hirst, 2006). En ese contexto, los procesos de regionalización y globalización caracterizaban el período que se abría con la caída de la Unión Soviética (Bernal-Meza, 2009). La regulación económica comenzó a transitar desde los Estados al ámbito de los bloques regionales. América Latina no estuvo exenta de la proliferación de estos procesos: en el subcontinente intentaron impulsarse algunas instancias de in-

1 Esta institución, creada en 1948, se constituyó como una primera instancia de carácter formal para la confluencia continental (Dos Santos, 2014). 
tegración desde diversas perspectivas.

Algunos autores (Briceño Ruiz 2014; Gudynas 2006; Sanahuja, 2012) concuerdan en que hasta la década del 2000, los procesos de integración estuvieron signados por los preceptos impulsados por el regionalismo abierto. Pero, a partir de 2005 con la consolidación de un nuevo ciclo político en la región, el regionalismo comenzó a ser repensado bajo un diseño 'post-liberal' (Da Motta y Ríos, 2007; Sanahuja, 2012) o 'post-hegemónico' (Briceño Ruiz, 2014; Tussie y Riggirozzi, 2012). Dentro de este nuevo regionalismo se inscriben la Alianza Bolivariana para los Pueblos de Nuestra América-Tratado de Comercio de los Pueblos y la UNASUR (Sanahuja, 2012), constituida en 2004 bajo el nombre de Comunidad Sudamericana de Naciones.

Las principales características de este nuevo regionalismo serían: (a) el retorno de la política a las relaciones exteriores y de las políticas de desarrollo; (b) la presencia del Estado en términos de política externa; (c) la persecución de mayor autonomía frente al mercado y el distanciamiento de Estados Unidos; (d) el impulso de proyectos que buscan crear instituciones y políticas comunes, profundizando la cooperación en términos no comerciales y el desarrollo de infraestructura regional con el objeto de reducir asimetrías; y (e) la búsqueda de dinámicas que incorporen la participación de actores no estatales, entre otras (Sanahuja, 2012).

En este marco surgieron nuevos liderazgos que buscaban conducir este nuevo proceso de integración regional. Frente a la alternativa histórica entre una tendencia de integración liderada por Estados Unidos y otra articulada en conjunto por los países de la región, comenzó a consolidarse una suerte de tercera vía impulsada por Brasil, de rasgos netamente sudamericanos (Bernal-Meza, 2009).

De esta forma, los proyectos subregionales impulsados por Brasil

han sido parte de su estrategia por mejorar su inserción en la estructura de poder económico y político mundial, dado el papel instrumental que ese país le asignó en el pasado a la integración re- gional sudamericana. Ese papel estaba asociado a la expansión de los mercados como mecanismo de profundización del desarrollo industrial y de apertura gradual de la economía nacional a la economía mundial (Bernal-Meza, 2013, s/p).

Esta tendencia daría cuenta de lo que Marini caracterizó como subimperialismo brasileño en la región y esta nueva estrategia permitiría dar un salto cualitativo al mismo proceso (Marini, 1970).

Tal es el caso del Mercado Común del Sur (MERCOSUR), fundado por Argentina, Brasil, Paraguay y Uruguay en 1991, cuya constitución estuvo signada por las reformas neoliberales que se impulsaron durante la década de 1990. En su nacimiento, contó con el auspicio de funcionarios, empresas multinacionales y capitalistas sudamericanos, y las grandes beneficiarias fueron las corporaciones de Brasil y Argentina, estableciendo un MERCOSUR de los negocios (Katz, 2006). Si bien fueron los gobiernos los que comandaron dicha iniciativa, los agentes más dinámicos dentro de este proceso fueron los grupos económicos locales -asociados con entidades bancarias locales y extranjeras, y vinculados a sectores como la industria agroalimentaria, la automotriz, la siderúrgica, la petroquímica, entre otras- y las empresas trasnacionales que se vincularon al bloque (Laredo, 1995). Tras la crisis que vivió durante el período de recesión, el MERCOSUR recobró fuerzas con el apoyo de las burguesías brasileñas y argentinas que se mostraban reticentes a la integración que planteaba el Área de Libre Comercio de las Américas (ALCA). Esta nueva etapa de rediseño regional estuvo vinculada a los cambios que se produjeron en numerosos países de Sudamérica con la llegada al poder de gobiernos que implementaron medidas neodesarrollistas, que permearon en el MERCOSUR.

El rol de Brasil en el marco de las negociaciones por el ALCA dio cuenta de su intento por preservar a las multilatinas de capitales brasileños, consideradas por el gobierno de ese país como sus Campeones Nacionales (Clemente, 2017). Tras las Cumbres de las Américas que se fueron realizando, los gobiernos de algunos 
países comenzaron a plantear sus disidencias en torno al cariz que tendría el acuerdo, en muchos casos presionados por las burguesías locales que se verían perjudicadas de avanzar el tratado. Las protestas 'por arriba' se entrelazaban con las resistencias populares 'por abajo' que se gestaban a partir de movilizaciones impulsadas por movimientos sociales y políticos, que señalaban los impactos negativos que el acuerdo generaría para la economía de los países latinoamericanos (Kan y Pascual, 2011). En la Cumbre de 2005, algunos mandatarios manifestaron su rechazo a constituir un área de libre comercio $y$, en este marco, fue central la imagen de un MERCOSUR integrado. Siguiendo a Julián Kan (2009, p. 5), durante los debates la

derrota del ALCA alejó a EE.UU. de la región, lo cual permitió que Brasil consolidara su liderazgo en la misma (...) Por otra parte, la derrota del ALCA generó espacio político para que nuevas instancias de integración de las que Brasil forma parte, como el Banco del Sur y la UNASUR, se desarrollen.

En este contexto es que surgió la UNASUR. Siguiendo a Briceño Ruiz (2010, p. 103),

El modelo que se adoptó en estas iniciativas de integración respondían en gran medida a la forma como Brasilia entendía el papel que América del Sur debía desempeñar en el mundo globalizado, una visión en buena medida matizada por los intereses nacionales brasileños.

Sin embargo, el gobierno de Hugo Chávez representó un contrapeso al liderazgo brasileño, imponiendo su visión en el marco de UNASUR de establecer una iniciativa que no solo impulsara la cooperación en términos económicos, sino también políticos y sociales, con la idea de establecer un bloque de poder. Según Bernal-Meza (2013), UNASUR poseía tres características que la diferenciaban de otras instancias. Por un lado, un pragmatismo ideológico y una flexibilidad comercial. Por otro lado, incluyó en la agenda conjunta temáticas de seguridad y defensa. Por último, evidenció capacidad política para resolver problemáticas intra-regionales, bilaterales y de política nacional.

Más allá de la incertidumbre que existe hoy, la UNASUR ha representado un motor importante de la IIRSA y, en ese marco, Brasil ha sido uno de los principales financiadores de algunas de las obras más importantes. En especial, ha apoyado aquellas que lo beneficiaban, ya sea porque favorecen su conexión con los mercados asiáticos o porque, mediante el financiamiento, pueden negociar con los países para obtener energía a bajo costo, que ayude a suplir su necesidad de abastecimiento energético.

\section{EL IIRSA, ¿CABEZA DE PLAYA DEL SUBIMPERIALISMO BRASILEÑO?}

Desde comienzos del siglo, Brasil comenzó a manifestar la necesidad de impulsar instancias de integración que tuviesen como eje Sudamérica, en lugar de Latinoamérica. Con la llegada de Lula Da Silva al gobierno en 2005, esta orientación se profundizó, poniendo el énfasis de la integración en tres ejes principales: seguridad y defensa, infraestructura y energía. Por un lado, se impulsó el desarrollo de infraestructura que acrecentara el potencial energético de la región y permitiera conectar las fuentes de energía a los principales centros de acumulación de capital. Esto permitiría a Brasil continuar creciendo sin tener que hacer frente a límites asociados a déficits energéticos. Por otro lado, se avanzó en la construcción y mejoramiento de carreteras y vías de conexión para favorecer la conexión brasileña con los mercados asiáticos en expansión (en especial, a partir de la irrupción de China en el mercado mundial desde su ingreso a la Organización Mundial de Comercio en 2001). En la actualidad, China es uno de los principales socios comerciales de los países latinoamericanos: un $80 \%$ de sus exportaciones y un $60 \%$ de sus importaciones se concentran en Brasil, Chile, México y Argentina (Bekerman, Dulcich y Moncaut, 2014). Entre 2007 y 2010, las exportaciones brasileñas obtuvieron un salto positivo de alrededor de 40 mil millones de dólares, de los cuales el 50\% fue por la profundización de las relaciones con China (Mansor de Mattos y Dias Carcanholo, 2011).

A través del Estado, el gran capital brasileño impulsó la necesidad de desarrollar instancias 
que promuevan la integración regional de América del Sur, señalando ante todo la importancia que dichas instancias tendrían para favorecer la acumulación de capital en ese territorio. De esa forma, ministerios y organismos públicos brasileños buscaron imponer una nueva hegemonía regional en tanto que "expresión de un amplio consentimiento, manifestado en la aceptación de las ideas y apoyado por recursos materiales y por instituciones" (Bieler y Morton, 2013, p. 24).

En 2008, la Agencia Brasileña de Desarrollo Industrial (ABDI), el Banco Nacional de Desarrollo Económico y Social (BNDES), el Ministerio de Hacienda y el de Desarrollo, Industria y Comercio presentaron el documento Política de Desarrollo Productivo. Innovar e invertir para sustentar el crecimiento, en donde plantean como una estrategia de la política de desarrollo la integración productiva con Sudamérica. Allí señalan que

el objetivo del programa es aumentar la articulación de las cadenas productivas y elevar el comercio con esa región, buscando ampliar la escala y la productividad de la industria doméstica. En este contexto, la integración de las infraestructuras logísticas y energéticas en América del Sur se muestra como un gran desafío y oportunidad. (ABDI et al., 2008, p. 5)

También, plantean la necesidad de posicionar a empresas de capitales brasileños entre los cinco principales jugadores de la región.

Por su parte, el Ministro de Relaciones Exteriores y jefe de la Secretaría de Asuntos Estratégicos durante la era Lula, Samuel Pinheiro Guimarães, publicó un documento en el que analiza los posibles escenarios para la región, así como las políticas que Brasil debería adoptar para promover el desarrollo:

El futuro de Brasil depende de América del Sur y el
futuro de América del Sur depende de Brasil (...)
La dificultad de contacto entre los países contri-
buyó, conjuntamente con las características de
su desarrollo y de su inserción en la economía
mundial, a hacer de América del Sur ese archipié-
lago de sociedades subdesarrolladas. (2010, s/p)

En julio de 2008, Lula creó la Secretaría de Asuntos Estratégicos (SAE), cuyas principales atribuciones son el planeamiento nacional a largo plazo de las opciones estratégicas y la articulación con el gobierno y la sociedad para formular políticas de desarrollo. En un documento de 2010, la SAE plantea que por las dimensiones económicas que posee Brasil, surgieron empresas de gran tamaño y muy competitivas hacia el exterior; las mismas deberían dirigirse en primer lugar hacia los países vecinos haciendo nuevas inversiones o adquiriendo empresas locales. Además, afirman que, por las características territoriales, demográficas y económicas, y por la política exterior adoptada en las últimas décadas, Brasil tiene una creciente importancia política en el plano internacional, tornándose actor indispensable en las negociaciones comerciales, ambientales, energéticas y de defensa. He aquí los dos rasgos característicos de una potencia subimperialista (Luce 2015; Marini 1970).

En este contexto, la IIRSA se transformó en una herramienta clave para Brasil. Empresas constructoras de capitales brasileños obtuvieron la licitación de numerosas obras y el BNDES financió gran parte de ellas, sobre todo las que tenían una importancia estratégica para el país.

\section{¿INFRAESTRUCTURA PARA INTEGRAR?}

La IIRSA fue presentada durante la Reunión de presidentes de América del Sur realizada en Brasilia en 2000. La misma tomó como plataforma un documento que había desarrollado Enrique Iglesias García -presidente del Banco Interamericano de Desarrollo (BID)- a pedido del presidente de Brasil, Cardoso. La idea principal que expresaba era que América del Sur sería una base de apoyo para la estrategia brasileña de obtener el reconocimiento como potencia mundial (López, 2012). La Iniciativa tenía por objetivo desarrollar infraestructuras en el área de Argentina, Bolivia, Brasil, Chile, Colombia, Ecuador, Guyana, Paraguay, Perú, Surinam, Uruguay y Venezuela. Las obras serían financiadas por organismos internacionales, tales como el BID, el Banco de Desarrollo de América Latina y el Fondo Financiero para el Desarrollo de los Países de la Cuenca del Plata, así como también por bancos como el BNDES. 
Brasil ha sido el país que más ha invertido en la IIRSA a través del BNDES, cuya estrategia para viabilizar proyectos se realiza a través de préstamos a empresas de capitales brasileños (Quispe, Loncharich, Espinoza y Mormontoy, 2013).

El papel del BNDES en la región se orientó (...) a la expansión de la base territorial del Brasil para la explotación de recursos naturales (...) Los ejes focalizan la competitividad externa regional [del capital brasileño] y no la generación de interdependencia entre los países. (Chiarella Quinhoes, 2011, p. 25)

La IIRSA no ha sido una iniciativa efectiva en términos de transformación productiva, sino que, partiendo desde una visión logística, tiene la perspectiva de explotar las potencialidades territoriales para conectar los centros extractores y productores de commodities -principalmente agroindustriales y minerales- con los mercados internacionales, promoviendo la especialización productiva y comercial, dependiente de la demanda externa y de bajo valor agregado (Chiarella Quinhoes, 2011).

Las principales beneficiarias de IIRSA han sido algunas multilatinas (Santiso, 2007). Entre ellas se destacan las constructoras Odebrecht, Queiroz Galvão, OAS y Andrade Gutiérrez (principales adjudicatarias de obras de IIRSA); las de telecomunicaciones Embratel y Telebras; las vinculadas a la energía Electrobras y Petrobras; y las de transporte, como Vale do Rio Doce (Durand, 2009). A su vez, empresas ligadas a la megaminería, al agronegocio y a la extracción hidrocarburífera serían ampliamente favorecidas con el desarrollo de las megaobras.

Algunas de estas empresas ligadas a la construcción e ingeniería desarrollaron numerosas obras entre las cuales se destacan la represa de Inambari y la carretera sobre el TIPNIS. Ambos proyectos son de suma importancia no solo por el peso en términos económicos que las obras tendrían para Brasil, sino también por las resistencias que se tejieron a su alrededor. Ambas son expresiones concretas de las tendencias y tensiones del subimperialismo brasileño.

\section{LA REPRESA DE INAMBARI}

Para garantizar el suministro de energía eléctrica, a través de acuerdos de algunos países de la región con Electrobrás, Brasil planteó la construcción de represas hidroeléctricas en Argentina, Bolivia, Colombia, Ecuador, Guayana Francesa, Guyana, Nicaragua, Perú, Surinam y Uruguay. Muchos de estos proyectos serían financiados por el BNDES y construidos por OAS y Odebrecht. Uno de los principales proyectos que el gobierno brasileño intentó impulsar fue la represa Inambari en Perú.

En abril de 2009, el presidente peruano Alan García, firmó un Memorando con Lula. El documento tenía por objetivo fortalecer las relaciones entre ambos países. Uno de los puntos centrales permitía que Brasil financie, construya y opere seis plantas hidroeléctricas en territorio peruano: Inambari, Sumabeni, Paquitzapango, Urubamba, Vizcatán y Chuquipampa. La mayor parte de la energía producida sería vendida a Brasil (Gil Mora, 2010).

La central de Inambari contaría con una línea de transmisión que la conectaría con las centrales del río Madeira en Brasil. El territorio donde se emplazaría se encuentra en la confluencia entre Madre de Dios, Cusco y Puno, donde convergen los ríos Araza e Inambari, contando con un área de inundación de 46 mil hectáreas (Ejatlas, sf).

En la región de Puno, se encuentra la represa de San Gabán, una hidroeléctrica de pasada (cuya característica principal es que no tiene una gran acumulación de agua corriente arriba de sus turbinas, sino que esta es movida por la corriente natural del cauce), la cual no produce inundaciones, así como tampoco el desplazamiento de población tal como el que genera una central de embalse. Esto llevó a que, al inicio, la población no considerara a Inambari una amenaza para el territorio. Sin embargo, al conocer el impacto que tendría, las resistencias comenzaron a surgir. Estas se articularon con la pelea de las personas que se dedicaban a la minería artesanal, que verían truncadas sus fuentes de ingreso. Estos sectores, a su vez, habían tenido enfrenta- 
mientos contra proyectos megamineros a gran escala. La lucha por el territorio se articuló con la batalla en defensa de la Carretera Interoceánica que conecta Puno y Madre de Dios con Brasil. Construida en 2004, fue una pelea de décadas de Ixs peruanxs de la zona por considerar que favorecería el comercio con Brasil. Una parte de la carretera estaría en el área de inundación de Inambari por lo cual sería necesario avanzar con un nuevo trazado. A su vez, la lucha en defensa del territorio que se vería afectado por Inambari se articuló con las resistencias que se tejieron en diferentes áreas del país ante el anuncio de las seis represas a construir, lo cual le dio un impulso más nacional y general al conflicto.

Ante el impacto socioambiental y el desplazamiento al que se verían forzadas miles de familias, en diciembre de 2009 se produjo una manifestación popular en la que se bloqueó durante dos días el puente que conecta Cusco, Puno y Madre de Dios. La protesta fue duramente reprimida (González Zamora, 2010). En marzo de 2010, el Frente de Defensa de Puno realizó una manifestación a la que se plegaron gremios, autoridades municipales y regionales, comerciantes y estudiantes de las localidades de Puno, Ayaviri, Juli y Yunguyo. En el documento que difundieron apuntaban que la Empresa de Generación Eléctrica Amazonas Sur (Egasur), integrada por OAS, Electrobrás y Furnas -subsidiaria de Electrobrás-, invertiría 4 mil millones de dólares en la construcción de la represa, que afectaría un total de 15 mil personas forzadas a emigrar y pondría en riesgo al Parque Nacional Bahuaja-Sonene (Zibechi, 2012).

En junio de 2010, los gobiernos de ambos países firmaron un acuerdo de entendimiento, en el que Perú se comprometía a exportar a Brasil los excedentes de energía que se produjeran en Inambari, estableciendo que los valores de venta se mantendrían fijos por treinta años. Se estimó que un $90 \%$ de la energía producida sería exportada hacia Brasil (Topovich, 2010). Era -sin dudas- un proyecto que expresaba la voluntad subimperialista de Brasil. Inambari, al igual que los otros cinco que se proyectaron, sería desarrollado por Electrobrás y beneficiaría a algunas empresas brasileñas quienes se involucrarían en la construcción, tales como Odebrecht, OAS y Andrade Gutiérrez.

En junio de 2011, el gobierno peruano anunció la conclusión y extinción de la concesión temporal atribuida a la Empresa de Generación Eléctrica Amazonas, arguyendo que la población no fue consultada previamente. Hasta hoy, la construcción de la represa continúa suspendida.

Durante el gobierno de Humala (2011-2016), hubo declaraciones que afirmaban la necesidad de reactivar proyectos suspendidos. Frente a esa posibilidad, la población de la región se mostró en estado de alerta por la posible reactivación de Inambari. Tras los escándalos de corrupción que ligaron a Odebrecht y OAS con políticos peruanos, el proyecto quedó enterrado.

\section{LA CARRETERA SOBRE EL TIPNIS}

Bolivia tiene una gran importancia en la IIRSA pues es considerada un "cuello de botella estratégico" por donde atraviesan cinco de los doce corredores planteados (Barreda, 2005). Uno de los principales proyectos es la carretera Villa Tunari-San Ignacio de Mojos, la cual conectaría los departamentos de Cochabamba y Beni, atravesando el TIPNIS (Territorio Indígena y Parque Nacional Isiboro-Secure), región donde habitan los pueblos indígenas mojeño, yurakaré y t'siman, agrupados en 64 comunidades de alrededor de 12 mil habitantes.

EI TIPNIS fue reconocido en 1990 tras la I Marcha por la Dignidad, por la Vida y por el Territorio, momento en el que pueblos indígenas de las tierras bajas y de la Amazonía se ubicaron como protagonistas en la escala nacional, y en el que el Estado boliviano reconoció la Convención 169 de la Organización Internacional del Trabajo. Además de las comunidades indígenas, otro grupo social clave, que habita en la región sur del TIPNIS, es el de los productores de coca, reconocidos por el Estado, desde hace poco tiempo, como comunidades interculturales (Porto-Gonçalves y Betancourt Santiago, 2013).

Este proyecto no es nuevo, dado que en la dé- 
cada de 1990 existía uno similar impulsado por intereses de empresas madereras y ganaderas. Sin embargo, desde 2003 la carretera integra el corredor bioceánico de IIRSA paralelo al bloque petrolero Sécure sobre el que la empresa Repsol adquirió los derechos de explotación por un período de 30 años (Zibechi, 2012).

La construcción de la carretera fue adjudicada a la constructora OAS, en un proceso muy cuestionado, ya que esta compañía fue la única proponente. A su vez, se planteó que el $80 \%$ del financiamiento sería otorgado por el BNDES (García Torres, 2011), el cual fue aceptado, en abril de 2011, por la Asamblea Legislativa de Bolivia. El proyecto implicaría la deforestación de miles de hectáreas, el cambio de cursos de agua, la afectación a drenajes naturales y la pérdida de la biodiversidad (Peñarrieta Venegas, 2007), además de los efectos negativos a nivel cultural para las comunidades allí emplazadas. La finalidad de la carretera, entre otras, está vinculada a la conexión Chile-Bolivia-Brasil mediante el desarrollo de un corredor bioceánico que una el Atlántico con el Pacífico (Ejatlas, sf).

Este proyecto, a su vez, se conecta con el modelo de desarrollo que buscó impulsar el Movimiento al Socialismo. Este modelo si bien estaba regido por numerosos aspectos que se planteaban desde movimientos sociales y en la nacionalización de algunos recursos considerados estratégicos, se centró en el desarrollo económico a través de un modelo primario exportador y extractivista.

En este contexto, las comunidades indígenas que habitan el TIPNIS llevaron adelante, entre agosto y septiembre de 2011, movilizaciones para oponerse al proyecto. En agosto se realizó la VIII Marcha rumbo a la ciudad de La Paz, la cual a pesar de ser duramente reprimida terminó con la movilización de 500 mil personas, que fueron atendidas, con algunas vacilaciones por el gobierno. Estas situaciones llevaron al quiebre del pacto indígena que llevó a Evo a la presidencia, sobre todo por la participación en las movilizaciones de las dos mayores organizaciones indígenas: la Confederación de Pueblos Indígenas del Oriente
Boliviano (CIDOB) y el Consejo Nacional de Ayllus y Markas del Qullasuyu (CONAMAQ) (Porto-Gonçalves y Betancourt Santiago, 2013). A su vez, otra gran movilización realizada el 28 de septiembre, fue duramente reprimida por la policía boliviana, dejando un saldo de 39 heridos.

A la par, los sectores ligados a la producción de coca también movilizaron, solo que, en este caso, contra la Ley $\mathrm{N}^{\circ} 180$ que declara la intangibilidad del TIPNIS. El gobierno de Morales no solo recibió a este sector, sino que, tomando sus demandas, aprobó la Ley $N^{\circ} 222$ de Consulta sobre la intangibilidad y la construcción de la carretera.

En febrero de 2012, el gobierno nacional promulgó la Ley de Consulta Previa a las comunidades indígenas del TIPNIS. El Informe Final de la Consulta determinó que 55 comunidades aceptaban la construcción y tres se oponían. Sin embargo, algunas comunidades comenzaron a denunciar la falta de transparencia de los resultados.

Ante las denuncias, del 29 de noviembre al 13 de diciembre de ese mismo año, representantes de la Asamblea Permanente de Derechos Humanos, la Federación Internacional de Derechos Humanos y la Red Pastoral Social Cáritas realizaron una verificación en el TIPNIS, llegando a la conclusión de que la consulta no había cumplido con los estándares internacionales necesarios, ni fue informada. Finalmente, concluyeron que 30 de las 35 comunidades rechazaban la construcción de la carretera (Peralta, 2017).

En julio de 2017, el gobierno envió a la Cámara de Diputados un proyecto de Ley de Protección, Desarrollo Integral y Sustentable del TIPNIS que establece que el desarrollo de caminos, carreteras, caminos y sistemas de navegación fluvial deberán realizarse de manera participativa con las comunidades. Por otro lado, establece pautas para el aprovechamiento con participación de privados. En agosto de ese año, la ley fue promulgada. Posteriormente, un grupo de diputados de la oposición presentaron ante el Tribunal Constitucional Plurinacional una acción de inconstitucionalidad, que hasta hoy no ha recibido respuesta. 
Del 14 al 25 de agosto de 2018, el Tribunal Internacional de Defensa de la Naturaleza visitó el TIPNIS. Los miembros de la comisión, Alberto Acosta (Ecuador), Tom Goldtooth (Estados Unidos), Shannon Biggs (Estados Unidos), Enrique Viale (Argentina) y Hana Begovic (Suecia), recogieron testimonios de integrantes de los pueblos indígenas que habitan la región, y a su vez gestionaron reuniones con el presidente $y$ con cocacoleros. En la sentencia final presentada por el Tribunal se consigna que la consulta realizada por el gobierno contó con múltiples irregularidades y que efectivamente la mayor parte de las comunidades rechazan el proyecto. A su vez, el Tribunal entendió que este proyecto violaría la Declaración Universal de los Derechos de la Madre Tierra y la Ley $N^{\circ} 071$ de Derechos de la Madre Tierra que rige en Bolivia desde diciembre de 2010.

Tanto el caso de la carretera como el de la represa Inambari sirven para ejemplificar los intereses que Brasil tiene en el diseño de la integración regional sudamericana para posicionar su liderazgo en la región y su peso a nivel internacional, pero también permiten dar cuenta de que el avance del subimperialismo supone conflictos $y$, más importante aún, de la capacidad que tienen las resistencias que se tejen en defensa de los territorios, pero también proponiendo modos diferentes de desarrollo, de integración y de vida.

\section{CONCLUSIONES PRELIMINARES}

El proyecto de la IIRSA expresa de manera mediada, conflictiva y contradictoria, los intereses del gran capital brasileño. A través de la misma se canalizan las tendencias subimperialistas que se han consolidado en el seno del ciclo del capital brasileño a través de cuatro décadas.

Esas tensiones surgen de la posición dependiente del capital brasileño en la configuración internacional del capital global. A través del IIRSA se busca compensar en el ámbito regional la pérdida de plusvalía que sufre la economía del Brasil frente al capital de los países imperialistas y que ha redundado históricamente en formas de super-explotación de la fuerza de trabajo local.
La dependencia de la economía brasileña construye a través de las tendencias subimperialistas una forma de integración que busca combinar el saqueo de las riquezas naturales de los pueblos de la región, con el proyecto de participación subordinada, pero en antagónica cooperación con las potencias del capital imperialista.

La resistencia de los pueblos de la región al avance de los megaproyectos de infraestructura, en particular a los proyectos de explotación energética, da cuenta del carácter contradictorio y disputado de los proyectos de integración regional liderados por el capital en sus distintas versiones históricas. Más allá de la centralidad que tienen esos proyectos para la reproducción ampliada del capital de la potencia hegemónica en la región en su vínculo con las potencias imperialistas (en especial, con Estados Unidos y -crecientemente- con China), el avance de los mismos de ninguna manera es materia definitiva. La más reciente avanzada, conducida con claridad por Brasil como potencia subimperialista, solo expresa una nueva forma en ese largo proceso histórico de integración regional 'por arriba'. Sin embargo, los proyectos de carretera en el TIPNIS y la represa de Inambari, y el rechazo de las poblaciones locales son muestra de ese proceso contradictorio que es el desarrollo capitalista, también en sus dimensiones infraestructurales. Por una parte, estos proyectos son centrales para la consolidación de la estrategia del capital brasileño en su proyección global. Por otro lado, sin embargo, ese avance violenta las condiciones materiales de vida de comunidades asentadas ancestralmente en los territorios. La modernización capitalista que se expresa en esos proyectos de infraestructura supone daños incalculables a esas poblaciones, no solo en las dimensiones económicas sino sobre todo en sus formas de vida; de ahí, la magnitud de las resistencias que provocan.

El futuro de la IIRSA como proyecto para la consolidación del sub-imperialismo brasileño está unívoca pero contradictoriamente atado a la capacidad de las comunidades en los territorios de la región para frenar la avanzada destructiva del capital. Esa capacidad está a 
su vez intrínsecamente vinculada al avance más general del capital subimperialista en toda la región. La crisis actual del capitalismo global y la crisis regional de los proyectos capitalis-

\section{REFERENCIAS}

ABDI, BNDES, Ministerio de Hacienda, y Ministerio de Desarrollo, Industria y Comercio. (2008). Política de Desarrollo Productivo. Innovar e invertir para sustentar el crecimiento.

Bambirra, V. (1995). El capitalismo dependiente latinoamericano. México: Siglo XXI.

Barreda, A. (2005). Geopolítica, recursos estratégicos y multinacionales. Curso Las Multinacionales españolas en América Latina, realizado en Bilbao, España.

Bekerman, M.; Dulcich, F. y Moncaut, N. (2014). La emergencia de China y su impacto en las relaciones comerciales entre Argentina y Brasil. Issue, 45(176), 55-82.

Bernal-Meza, R. (2009). El Regionalismo: conceptos, paradigmas y procesos en el sistema mundial contemporáneo. Revista Aportes para la Integración Latinoamericana, XV(21), 1-29.

Bernal-Meza, R. (2013). Modelos o esquemas de integración y cooperación en curso en América Latina (UNASUR, Alianza del Pacífico, ALBA, CELAC): una mirada panorámica. Ibero Online, 12.

Bieler, A. y Morton, A.D. (2013). Hegemonía, orden mundial y cambio histórico: siguiendo el camino de la teoría crítica. Perspectivas neogramscianas en las relaciones internacionales ( $1^{\circ}$ edición, pp. 23-42). En J. Kan y R. Pascual (Comps.) Integrados (?). Debates sobre las relaciones internacionales y la integración regional latinoamericana y europea. Buenos Aires: Imago Mundi.

Briceño Ruiz, J. (2010). La UNASUR: ¿Continuum o un nuevo inicio del regionalismo sudamericano? ( $1^{\circ}$ edición, pp. 103-21). En L. Martínez Alfonso, H. Ramanzini Júnior y $\mathrm{M}$. Vázquez (Eds.) Anuario de la Integración Regional de América Latina y el Gran Caribe. Buenos Aires: Coordinadora Regional de Investigaciones Económicas y Sociales. tas nacionales se expresan en una parálisis del proyecto IIRSA y abre en este plano una nueva etapa de conflictos por el futuro del control del territorio y las formas de vida.

Briceño Ruiz, J. (2014). O regionalismo latino-americano depois do regionalismo aberto: novos eixos, novas estratégias, modelos diversos ( $1^{\circ}$ edición, pp. 193-238). En C. Alves do Carmo, É. Winand, I.R.Barnabé y L. Miranda Pinheiro (Orgs.) Relações Internacionais Olhares cruzados. Brasilia: Fundação Alexandre de Gusmão.

Caffentzis, G. (1980). The Work/Energy Crisis and the Apocalypse (Vol. 1). Midnight Notes. Recuperado de https://libcom.org/library/workenergy-crisis-apocalypse-george-caffentzis

Caffentzis, G. (2013). In Letters of Blood and Fire: Work, Machines, and the Crisis of Capitalism. PM Press: Common Notions.

CEPAL. (1994). El regionalismo abierto en América Latina y el Caribe. La integración económica al servicio de la transformación productiva con equidad. Santiago de Chile: CEPAL.

Chiarella Quinhoes, R. (2011). Redes y territorio: la Iniciativa IIRSA en foco. Espacio y Desarrollo, 23, 5-29.

Cielo, C. y Vega, C. (2015). Reproducción, mujeres y comunes. Leer a Silvia Federici desde el Ecuador actual. Nueva Sociedad, 256, 132-144.

Cleaver, H. (1985). Una lectura política de «El Capital». Buenos Aires: Fondo de Cultura Económica.

Clemente, D. (2017). Las estrategias IIRSA-COSIPLAN y "Campeones Nacionales" en el proyecto regional de Brasil: la construcción de una hegemonía (2002-2016). Tesis de Maestría. Facultad Latinoamericana de Ciencias Sociales, Buenos Aires, Argentina.

Clemente, D. (2018). Los aportes de Ruy Mauro Marini a los estudios internacionales desde América Latina. Análisis político, 94, 75-92.

Da Motta, P. y Ríos, S. (2007). O Regionalismo pós-liberal na América do Sul: origens, iniciativas e dilemas. Serie comercio internacional, 62, 5-48. 
de Arruda Sampaio Jr., P. (2008). Notas sobre los desafíos de la integración latinoamericana (1o edición, pp. 30-64). En O. Martínez (Ed.) La integración en América Latina: de la retórica a la realidad. La Habana: Ruth Casa Editorial.

de Medeiros, C.A. (1986). Industrialización y desarrollo en el Brasil. Perspectivas y ajuste (1ºdición, pp. 108-144). En E. Torres Rivas y E. Deutscher (Eds.) Industrialización en América Latina. Crisis y perspectivas. San José: Facultad Latinoamericana de Ciencias Sociales y Fundación Friedrich Ebert.

Dos Santos, T. (2014). Economía mundial. La integración latinoamericana. México: Plaza \& Janes.

Durand, F. (2009). El eje Lima-Brasilia (donde algunos entran en arcos y salen con flechas). Nueva Sociedad, 219, 113-26.

Environmental Justice Atlas (Ejatlas) (sf). Ejatlas. Recuperado de https://ejatlas.org

Fajnzylber, F. (1990). Industrialización en América Latina: de la "caja negra" al "casillero vacío". Comparación de patrones contemporáneos de industrialización. Santiago de Chile: CEPAL.

Federici, S. (2018). El patriarcado del salario. Críticas feministas al marxismo. Madrid: Traficantes de Sueños.

Féliz, M. y Haro, A.C. (2019). Dependencia, valor y naturaleza. Hacia una revitalización crítica de la teoría marxista de la dependencia. Revista Sociedad, 38, 45-56.

Féliz, M. y Díaz Lozano, J.A. (2018). Trabajo, territorio y cuerpos en clave neodesarrollista. Argentina, 2002-2016. Revista Perfiles Latinoamericanos, 26(52), 1-26.

Féliz, M. y Migliaro, A. (2017). Desigualdad en sociedades extractivistas: Intersecciones de clase, género y territorio en el neodesarrollismo. (In)Justicias espaciales en Argentina y América Latina. Buenos Aires: Universidad Nacional de Rosario.

García Torres, M. (2011). Proyectos de infraestructura vial en la Amazonía: procesos, actores, intereses e impactos asociados. Estudio de caso: carretera Villa Tunari-San Ignacio de Mojos. Revista El Ecologista, 71, 41-43.

Gil Mora, J.E. (2010). Represa Inambari: importan- cia e impactos ambientales. Tampa: University of South Florida.

Gil, L. y Paikin, D. (2013). Mapa de la Integración Regional en América Latina. Procesos e instituciones. Nueva Sociedad, 9, 1-34.

González Zamora, A. (2010). Represión policial por hidroeléctrica Inambari. En Chaski (blog).

Gudynas, E. (2006). Los fantasmas de la integración regional. Revista del Sur, 166, 3-25.

Hirst, M.(2006). Los desafíos de la política sudamericana de Brasil. Nueva Sociedad, 205, 31-40.

Kan, J. y Pascual, R. (2011). Integración regional en América Latina y Argentina: ¿Solamente una cuestión de Estados? ( $1^{\circ}$ edición, pp. 45-79). En A. Bonnet (Comp.) El país invisible. Debates sobre la Argentina reciente. Buenos Aires: Peña Lillo y Ediciones Continente.

Kan, J. (2009). Discutiendo el liderazgo de Brasil en el contexto actual de la integración regional. XXVII Congreso de la Asociación Latinoamericana de Sociología y VIII Jornadas de Sociología de la $U B A$. Buenos Aires, Argentina.

Katz, C. (2006). El rediseño de América Latina. ALCA, MERCOSUR y ALBA. Buenos Aires: Ediciones Luxemburg.

Laredo, I. (1995). Proyecto MERCOSUR y grupos económicos en la Argentina: posiciones y debates. Santa Fe: Universidad Nacional del Litoral.

López, R. (2012). Nuevo instrumento de regionalismo sudamericano. Resultados de la Iniciativa para la Integración de la Infraestructura Regional Sudamericana (IIRSA) (pp. 217-37). En R. Bernal-Meza y S. Quintanar (Orgs.) Regionalismo y Orden Mundial: Suramérica, Europa, China. Buenos Aires: Nuevo Hacer, UNICEN.

Luce, M. (2007). O subimperialismo brasileiro revisitado: a política de integração regional do governo Lula (2003-2007). Tesis de Maestría. Universidade Federal do Rio Grande do Sul, Brasil.

Luce, M. (2015). Sub-imperialism, the highest stage of dependent capitalism (pp. 27-44). En P. Bond (Ed.) BRICS. An Anti-Capitalist Critique. Londres: Pluto Press.

Luxemburg, R. (2018). La acumulación de capital. Edicions internacional Sedov. Germinal - en 
defensa del marxismo.

Mansor de Mattos, F.A. y Dias Carcanholo, M. (2011). Amenazas y oportunidades del comercio brasileño con China: lecciones para Brasil. Problemas del Desarrollo, 43(168), 117-145.

Marini, R.M. (1970). Subdesarrollo y revolución. México: Siglo veintiuno editores.

Marini, R.M. (1977). La acumulación capitalista mundial y el sub-imperialismo. Cuadernos Políticos, 12, 21-39.

Marini, R.M. (2015). América Latina, dependencia y globalización / Ruy Mauro Marini. Buenos Aires: Siglo XXI y CLACSO.

Osorio, J. (2017). Ley del valor, intercambio desigual, renta de la tierra y dependencia. Argumentos, 30(83), 219-48.

Peñarrieta Venegas, L. (2007). Evaluación económica de la construcción de la carretera ViIla Tunari - San Ignacio de Moxos, dentro el Territorio Indígena y Parque Nacional Isibóro Sécure (TIPNIS). Conservación Estratégica, 5.

Peralta, P. (2017). Las nueve preguntas clave sobre el conflicto del TIPNIS. Página Siete (blog). Recuperado de https://www.paginasiete.bo/ nacional/2017/8/14/nueve-preguntas-clave-sobre-conflicto- tipnis-148246.html

Pinheiro Guimarães, S. (2010). A América do Sul em 2022. Carta Maior.

Porto-Gonçalves, C.W. y Betancourt Santiago, M. (2013). Encrucijada latinoamericana en Bolivia: el conflicto del TIPNIS y sus implicaciones civilizatorias. La Paz: Autodeterminación.

Quispe, L.; Loncharich, I.; Espinoza, L. y Mormontoy, E. (2013). La integración en infraestructura sudamericana: un análisis a partir del liderazgo regional brasileño. Revista Andina de
Estudios Políticos, III(1), 166-85.

SAE. (2010). Brasil 2022: Trabajos preparatorios. Brasilia: SAE y Presidência da República. Recuperado de https://www.cartamaior. com.br/?/Editoria/Internacional/A-America-do-Sul-em-2022/6/15963

Sanahuja, J.A. (2012). Regionalismo post-liberal y multilateralismo en Sudamérica: El caso de UNASUR (1 ${ }^{\circ}$ edición, pp. 19-71). En A. Serbin, L. Martínez, y H. Ramanzini Júnior (Eds.) El regionalismo "post-liberal" en América Latina y el Caribe: Nuevos actores, nuevos temas, nuevos desafíos. Buenos Aires: CRIES.

Santiso, J. (2007). The Emergence of Latin Multinationals. Frankfurt: Deutsche Bank Research.

Topovich, E. (2010). Mayor parte de energía irá a territorio brasileño. Diario La Primera. Recuperado de https://www.diariolaprimeraperu. com/online/politica/mayor-energia-ira-territorio-brasileno-63242/

Tussie, D. y Riggirozzi, P. (2012). The Rise of Post-hegemonic Regionalism. Netherlands: Springer.

Zibechi, R. (2012). Brasil Potencia. Entre la integración regional y un nuevo imperialismo. Bogotá: Ediciones Desde Abajo.

\section{OTRAS FUENTES}

Ley Nacional $N^{\circ} 71$ de Derechos de la Madre Tierra (2010).

Ley Nacional No 180 de Protección del Territorio Indígena y Parque Nacional Isiboro Sécure - TIPNIS (2011).

Ley Nacional N²22 de Consulta a los Pueblos Indígenas del Territorio Indígena y Parque $\mathrm{Na}$ cional Isiboro Sécure - TIPNIS (2012).

Mariano Féliz es Dr. en Economía y Dr. en Ciencias Sociales. Magister en Sociología Económica. Lic. en Economía. Es docente-Investigador categoría II. Consejero Directivo del CIG-FaHCE/UNLP. Integrante de la colectiva Al Borde (Construyendo Pensamiento Indisciplinado), de la Sociedad de Economía Critica (SEC) y del Comité Editorial de Cuadernos de Economía Crítica. Es editor de la serie de libros Critical Studies on Latin America. Debates and Alternatives for Social Change (Editorial Ibidem, Alemania). Ha publicado artículos en Latin American Perspetives, Review of Radical Political Economics, Historical Materialism, Desarrollo Económico y Theomai, entre otras. Ha escrito y compilado 
varios libros. Sus temas de investigación abarcan la teoría marxista de la dependencia, macroeconomía del desarrollo y la economía política marxista. Ha realizado estancias de investigación en Francia y Venezuela, y dictado conferencias en espacios académicos de Alemania, Inglaterra, y varias universidades de Argentina, Uruguay y Brasil. Centro de Investigaciones Geográficas (CIG). Instituto de Investigaciones en Humanidades y Ciencias Sociales (IdIHCS/CONICET). Universidad Nacional de La Plata (UNLP). Calle 51 e/124 y 125. Oficina C313-C315, Edificio C (1925) Ensenada, Buenos Aires, Argentina, marianfeliz@gmail.com, ORCID https://orcid.org/0000-0002-3000-3873

Daiana Melón es Doctoranda en Ciencias Sociales (UNLP). Maestranda en Gobierno (UBA). Lic. en Comunicación Social (UNLP). Becaria doctoral de la UNLP. Integrante de la colectiva Al Borde (Construyendo Pensamiento Indisciplinado). Ha publicado artículos en Ecología Política, Lutas Sociais y Onteaiken, entre otras. Ha coordinado el libro La Patria Sojera. El modelo agrosojero en el Cono Sur (Editorial El Colectivo) y ha escrito capítulos en algunos libros. Sus temas de investigación abarcan los conflictos socioambientales, la teoría marxista de la dependencia y la integración regional. Ha realizado una estancia de investigación doctoral en España y dictado un taller en la Universidad de la República de Uruguay. Centro de Investigaciones Geográficas (CIG). Instituto de Investigaciones en Humanidades y Ciencias Sociales (IdIHCS/CONICET). Universidad Nacional de La Plata (UNLP). Calle 51 e/124 y 125. Oficina C313-C315, Edificio C (1925) Ensenada, Buenos Aires, Argentina, daianamelon@gmail.com, ORCID https://orcid.org/0000-0003-2074-0201 